\title{
Sensibilización Ambiental en Educación Básica: Una Experiencia de Aprendizaje para Abordar la Sustentabilidad utilizando el Diseño y la Ciencia Ficción
}

\author{
Samuel Soto(1) Juan C. Briede(1), Marcela L. Mora(2) \\ (1) Universidad del Bío-Bío, Departamento de Arte y Tecnologías del Diseño, \\ Avda. Collao 1202, Casilla 5-C, Concepción-Chile (e-mail: ssoto@ubiobio.cl; jbriede@ubiobio.cl). \\ (2) Universidad del Bío-Bío, Área de Desarrollo Pedagógico y Tecnológico, Coordinación Unidad de \\ Gestión Curricular y Monitoreo, Avda. Andrés Bello s/n, Casilla 447, Chillán-Chile (e-mail: \\ mamora@ubiobio.cl).
}

Recibido Jun. 1, 2016; Aceptado Ago. 3, 2016; Versión final Oct. 5, 2016, Publicado Abr. 2017

\begin{abstract}
Resumen
Se presentan los resultados de una experiencia educativa que abordó desde el diseño la importancia de la sustentabilidad para tratar problemas medioambientales. Utilizando el recurso narrativo de la ciencia ficción para contextualizar prospectivamente e inspirar a las alumnas en la solución de problemáticas globales con el recurso agua. Se implementó un piloto de feria multimodal y se aplicó una encuesta de percepción a 106 estudiantes de octavo básico en Talcahuano, Región del Biobío, Chile. Los resultados muestran que 97\% de los estudiantes terminó asignando importancia a la sustentabilidad, $81 \%$ a la necesidad de reciclar y ese mismo porcentaje expone que el uso de la ciencia ficción para la creación de sus productos tendientes a extracción y reutilización del recurso agua, fue una experiencia que determinó satisfactoriamente su participación. De esta manera fue posible detectar que la integración multimodal de la experiencia permitió motivar y captar la atención de las alumnas durante la actividad, además de ampliarles los campos de aplicación del diseño.
\end{abstract}

Palabras clave: diseño industrial, educación tecnológica, conciencia ecológica, sustentabilidad, reciclaje, reutilización, creatividad

\section{Environmental Awareness in Basic Education: A Learning Experience to Approach Sustainability using Design and Science Fiction}

\begin{abstract}
The results of an educational experience that addressed from the design the importance of sustainability to deal with environmental problems are presented. Using the narrative resource of science fiction to contextualize prospectively and inspire the students in the solution of global problems regarding water resource. A pilot of multimodal fair was implemented and a perception survey was applied to 106 eighth grade students in Talcahuano, Biobío Region, Chile. The results show that $97 \%$ of the students ended up assigning importance to sustainability, $81 \%$ to the need of recycling and the same percentage states that the use of science fiction for the creation of their products for extraction and reuse of water resources was an experience that satisfactorily determined their participation. In this way it was possible to detect that the multimodal integration of the experience allowed motivating and capturing the attention of the students during the activity, in addition to extending the fields of application of the design.
\end{abstract}

Keywords: industrial design, technology education, environmental awareness, sustainability, recycling, reuse, creativity 


\section{INTRODUCCIÓN}

Las reflexiones sobre la problemática ambiental global, después de tres décadas desde la Conferencia de las Naciones Unidas sobre el Medio Humano, celebrada en Estocolmo, plantea que la única manera de detenerla es mediante un cambio radical del modo de relacionarnos con nuestro entorno. Junto con ello, la sobre-explotación de recursos del planeta por un tercio de la población mundial opulenta y despilfarradora y dos tercios que se debaten entre la miseria y la muerte, resulta una paradoja que la educación ambiental intenta detener (Cuello, 2003). En este marco se identifica la necesidad de abordar la problemática ambiental desde escenarios educativos, con el fin de transformarla en un activo significativo a partir de experiencias que apelen a la sensibilidad y toma de conciencia de los individuos. Se espera, introducir un poder de decisión en las actividades cotidianas que permitan un cuidado por el medio ambiente desde su propia realidad, y para para lograrlo, se han explorado diversos formatos y estrategias, pasando desde instancias informativas -donde el receptor es pasivo- hasta instancias como ferias con talleres donde los asistentes se implican activamente en el desarrollo y aplicación.

Así la Carrera de Diseño Industrial de la Universidad del Bío-Bío, preocupada de éste problema ambiental local y global, específicamente con respecto al recurso natural agua, desarrolló un proyecto de extensión basado en una feria multimodal cuyo objetivo fue la indagación acerca de la importancia del reciclaje o reutilización para ayudar a la sustentabilidad, utilizando la ciencia ficción como recurso didáctico narrativo, que permitiera conducir los procesos de contextualización prospectiva de la problemática planteando la problemática del recurso agua en el planeta Marte. La exposición multimedial busca contextualizar inmersivamente realidad marciana. El equipo docente se encargó del diseño y montaje de la exposición, así como de la guía didáctica para ser entregada a las alumnas, que contiene las directrices para desarrollar el desafío de diseño. Por su parte las alumnas debieron elaborar propuestas/maquetas de soluciones a partir del "reciclado" de envases de alimentos que ellas habían consumido.

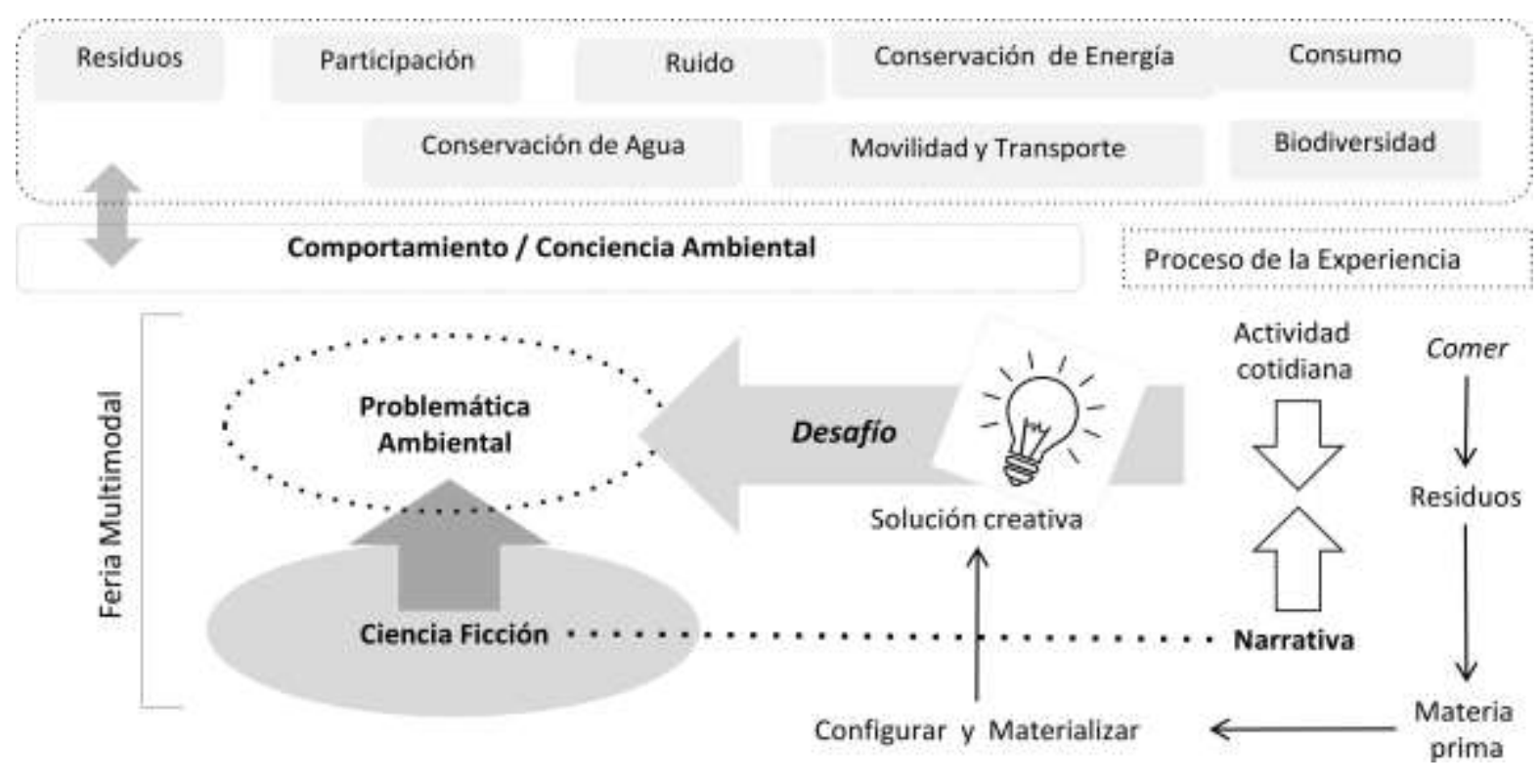

Fig. 1: Comportamiento Ambiental. Adaptado de Stern et al., (1999).

\section{Sustentabilidad y Educación}

Una estrategia para sensibilizar y generar conductas arraigadas en las futuras generaciones es abordar el tema de la sustentabilidad y cuidado del medio ambiente en la educación escolar. La educación no solo aumenta el acceso a fuentes y tipos de información, sino que también puede conducir a una mejor comprensión de mensajes ambientales complejos como el cambio climático (McCright, 2010).

Para establecer la relación causal entre la educación y los comportamientos verdes, Chankrajang y Muttarak (2017) estudiaron la relación entre la escolarización /educación formal y las conductas proambientales. Los resultados arrojaron que más años de escolarización conducen a una mayor probabilidad de llevar a cabo acciones basadas en el conocimiento y respetuosas con el medio ambiente, pero no de ahorro de costos en acciones pro-ambientales. Investigaciones acerca de la conciencia de la sostenibilidad (Olsson y Gericke, 2016) han demostrado que el interés y preocupación por los problemas ambientales 
tiende a disminuir en la adolescencia, lo que sugiere que es clave poder abordarlos a temprana edad. Considerando que los niños tienen la capacidad de adoptar roles activos como ciudadanos y son contribuyentes cruciales a las exploraciones de las prácticas de consumo contemporáneas y un futuro sostenible, aunque generalmente no se anima explícitamente a los niños a participar activamente en la construcción de discursos de consumo sostenible. La investigación de Donovan (2016) demuestra la aplicación de narrativa visual como medio para que los niños puedan construir narraciones sobre el consumo sostenible (Donovan, 2016).

Uno de los modelos que permite abordar este problema es el Modelo de Comportamiento Ambiental de Stern (1999), que corresponde a un modelo teórico de Valor-Creencia-Norma, (Value-Belief-Norm Model, VBN) de la segunda perspectiva teórica. Lo constituyen variables, tales como: Valores (Biosférico, Altruista y Egoísta); Creencias (medidas a través del nuevo paradigma ecológico o visión ecológica); Conciencia de las Consecuencias y Atribución de Responsabilidad y Normas Personales, considerando éstas como el sentimiento de obligación de llevar a cabo un comportamiento ambiental responsable según las dimensiones que la Fig. 1 presenta. Además de aplicar un modelo teórico el que permita la comprensión del problema ambiental.

\section{Ciencia ficción y la enseñanza}

La ciencia ficción está orientada a explorar y plantear aspectos imposibles o situaciones ideales a un tiempo determinado. La ficción tiene la capacidad de identificar y evaluar conceptos y tecnologías innovadores, conformar campos de desarrollo como la genómica, la clonación y la co-constitución de la innovación (Bassett, et al., 2013). Robinson et al. (2013) sugiere que la ficción ayuda a identificar qué investigación es necesaria en términos de nuevos conocimientos, técnicas y materiales. Ofrece formas de encarnar, contar, imaginar y simbolizar 'futuros', que pueden proporcionar marcos y entendimientos alternativos para enriquecer los grandes retos del siglo XXI (Binaa et al., 2016) y generar estrategias para abordarlos.

Dentro de las posibles soluciones para abordar los problemas medio ambientales, respecto a la población y/o recursos, el foco está puesto en la colonización espacial. Es así como Marte, el planeta rojo, ha sido fuente de numerosos estudios respecto al recurso agua en la superficie planetaria (Pála y Kereszturib, 2017) y considerar las primeras misiones humanas a marte para el 2035 para lo cual la NASA está desarrollando a largo plazo tecnologías clave necesarias para estas misiones (Moore, 2010), ya que tomará muchos años para que lleguen a la madurez.

La ciencia ficción y su relación con la enseñanza, tanto como género narrativo, así como recurso didáctico se puede evidenciar ya por ejemplo en el trabajo realizado por Thoring y Mueller (2012) en el cual se utilizaron las historias del autor de ciencia ficción Philip K. Dick como fuente de inspiración para el diseño de productos imaginarios considerando el escenario y contextualización socio-técnico.

Brake, M., \& Thornton (2003) creen que el uso de fragmentos de películas para enseñar la ciencia puede reforzar el interés del estudiante en la ciencia, sobre todo debido al alto grado de relación entre estas películas y la vida cotidiana de los estudiantes. Además, Laprise y Winrich (2010) también han tratado de utilizar las películas de ciencia ficción para generar el interés de los estudiantes en el aprendizaje de la ciencia. El estudio de Blythe y Wright (2006) se centró en la integración de la ciencia ficción y las películas, en las actividades de educación de diseño de tecnología para estimular la imaginación del estudiante a través de las parcelas de ficción de la ciencia ficción o el cine y la posterior aplicación de la imaginación a las ideas de mejora de productos de uso diario.

\section{El Diseño y la resolución de problemas}

El diseño, entendido como la resolución de problemas, es una forma natural y la más ubicua de las actividades humanas (Braha y Maimon, 1997):"Este proceso comienza con el reconocimiento de las necesidades y la insatisfacción con el estado actual de las cosas, y la comprensión de que alguna acción debe llevarse a cabo con el fin de resolver el problema" que implica la adquisición, evaluación, producción y transferencia de conocimientos específico (Woelfel et al., 2010).

El diseño de productos, como disciplina proyectual tradicionalmente se ha abordado mediante una serie de metodologías (Manzini, 1993) y procesos proyectuales (Jones, 1977) centrados en la obtención de productos y objetos. Hoy en día, con cada vez más urgencia, se hace necesario considerar y resolver los problemas medioambientales derivados de la producción y la generación de desechos que dañan al planeta. Estrategias como el ecodiseño (Capuz et al., 2002), buscan desarrollar productos desde un enfoque sustentable, constituyéndose en un parámetro de diseño dentro del proceso metodológico (Birkhofer, 2011) aplicando un modelo 'de la cuna a la cuna' (Braungart y McDonough, 2005), que 
contempla la integración de valores y cualidades sustentables no solo en las fases de fabricación y desarrollo sino desde el proceso de ideación hasta el desecho, considerando la sostenibilidad ambiental durante todo su ciclo de vida (Vallet, et al., 2013). Considera una gran variedad de enfoques, métodos y herramientas que ofrecen a los diseñadores un enfoque estructurado para integrar criterios ambientales en el proceso de diseño y presentan diferentes objetivos (Birch et al., 2012) enfocados principalmente en la creación de valor local dentro del eco diseño (Tyla et al., 2015). Siendo la percepción de estos atributos físicos por parte del consumidor un aspecto clave a la hora de poder identificarlos y adquirirlos (Diego-Mas, Poveda-Bautista, Alcaide-Marzal, 2016).

\section{METODOLOGÍA}

El desarrollo del proyecto se abordó considerando dos etapas distintivas; la primera relacionada con el proceso de ideación y generación del marco conceptual, para definir el carácter de los elementos que circunscriben la historia y la segunda relacionada con la percepción de las estudiantes respecto a la actividad y a los aspectos y contenidos abordados en ella.

\section{Primera etapa: Definición temática y diseño de exposición}

i) Definición de guion temático: El equipo de docentes realiza la definición de algunos elementos claves se logra contextualizar el elemento a desarrollar (contexto geográfico, económico, social y tecnológico). Además de la preparación de la guía Pedagógica en la cual se presentan las directrices que las alumnas seguirán para desarrollar las propuestas

ii) Diseño/Construcción de Dioramas con Naves: (Figura 2)

(a) Ideación y bocetado de naves: En base a la historia planteada se generan las primeras ideas de las naves marcianas a través del uso de bocetos; (b) Modelado tridimensional de las naves: Se definen partes y piezas del sistema; (c) Reutilización y reciclaje: Diseño de detalles: Se definen las partes y piezas del prototipo considerando partes y piezas de productos electrónicos en desuso. Por ejemplo ventiladores de computadores como rotores de la nave. Carcaza de galletera como habitáculo de la nave; (d) Fabricación de prototipos: Se construyen prototipos considerando las estrategias de ecodiseño propuestas por Brezet \& Van Hemel (1997) y el primer nivel de componente del producto, en las cuales se define el reciclaje y la reutilización (de partes y piezas de algún producto previamente manufacturado). Se generan propuestas conceptuales, luego modelos tridimensionales y naves realizados a partir de reciclaje y reutilización de artefactos. (Figura 2); (e) Acabado superficial: Pintado, instalación de carcaza; y (f) Dioramas: Se construyen las bases expositivas sobre las cuales van instaladas las naves. Para lo cual se generan superficies irregulares que simulen la superficie del Planeta Marte, mediante el uso de colores y texturas.

iii) Diseño y diagramación de infografías: (Figura 3)

1.1 Diseño de experiencia de aprendizaje: Desafío. Reciclaje y reutilización como elemento clave. Reconfigurar los envases de deshecho para generar nuevas propuestas que den respuesta a las problemática planteada. Exposición (Figura 4), Colación, Encargo y desarrollo (Figura 5), Presentación de Proyectos y Premiación (Figura 6).

\section{Segunda etapa: Estudio de la experiencia de aprendizaje}

Según los modelos y recursos (tanto digitales como análogos) y considerando el espacio y las condiciones espaciales de la sala del establecimiento educacional es que se diagrama la organización de la exposición. Se busca generar recursos gráficos y tridimensionales desarrollando tanto los paisajes evocativos en gigantografías, tal como se puede apreciar en la figura 2, así como los modelos físicos que son parte de los dioramas en la Figura 3. Estos dioramas permiten situar y contextualizar en un espacio físico las maquetas de naves construidas previamente y apoyar las tres instancias de la experiencia: Exposición, Desafío, Premiación.

El Recurso de la ciencia ficción se utiliza como discurso narrativo para contextualizar "dramáticamente" una gran problemática; buscando impresionar, seducir y motivar a los estudiantes para que se comprometan en la actividad. Del punto de vista del diseño a nivel macro, los alumnos son protagonistas del proceso de resolución de problemas. Identificando el problema, sus factores claves y luego la propuesta de diseño que va a responder a este contexto y sus variables. 


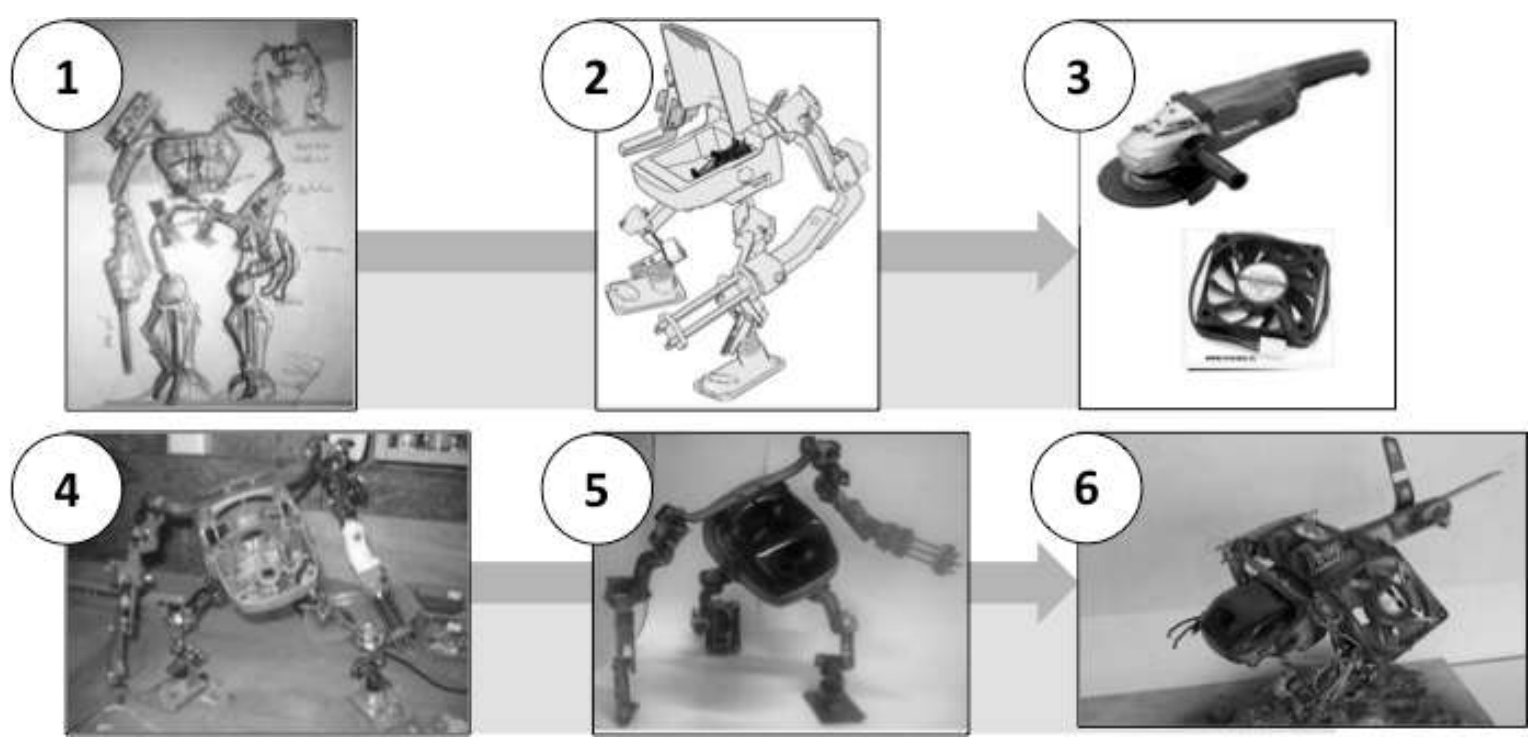

Fig. 2: Secuencia diseño y elaboración de naves espaciales.

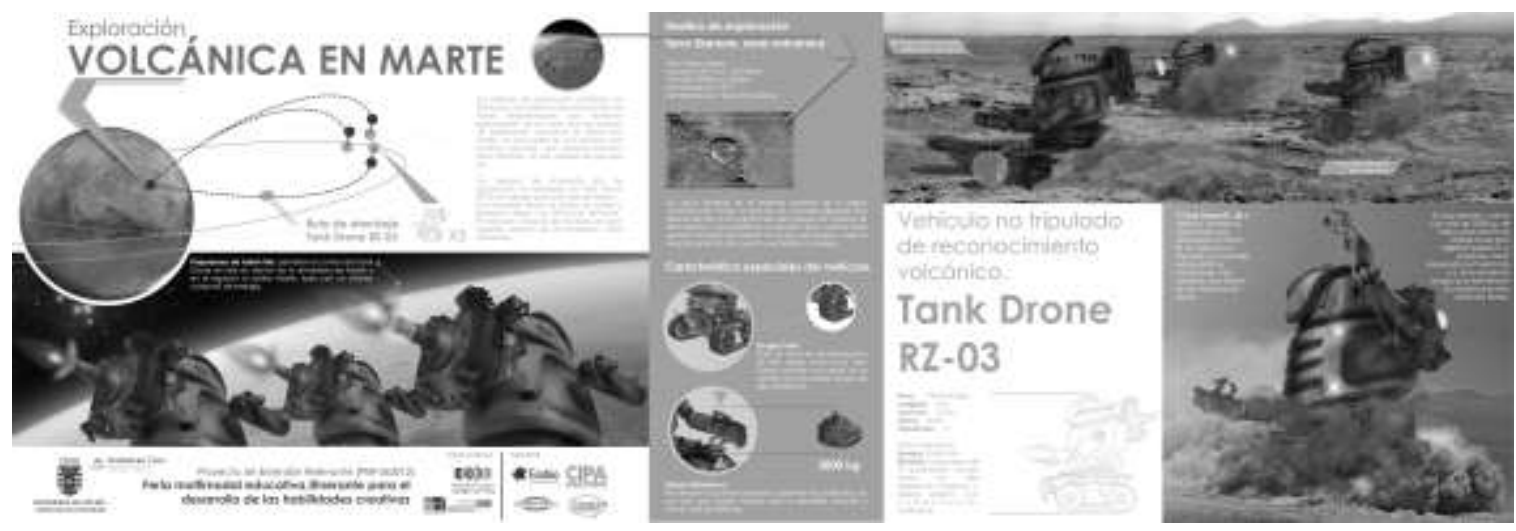

Fig. 3: Composición Infografía de Marte.

La historia de ciencia ficción otorgó las características del contexto (Planeta Marte) y la necesidad de recoger, almacenar y distribuir el recurso hídrico que es muy escaso y considerando la anatomía y modos particulares de los alienígenas. Por tanto las soluciones buscaban responder a sistemas de recogida, por ejemplo: vehículos colectores, contenedores y tanques transportables, o cámaras de escaneo para identificación de yacimientos en la geografía marciana.

Tabla1: Distribución de alumnas y asignación de materiales.

\begin{tabular}{|c|c|c|c|}
\hline $\begin{array}{c}\text { Universo total de } \\
\text { participantes }\end{array}$ & $\begin{array}{c}\text { Número de alumnas por } \\
\text { grupo }\end{array}$ & Envases por alumna & $\begin{array}{c}\text { Materiales e insumos por } \\
\text { grupo }\end{array}$ \\
\hline & & & Tijeras \\
& & $\begin{array}{l}\text { Cinta de enmascarar } \\
1 \text { envase de jugo pequeño }\end{array}$ & $\begin{array}{l}\text { Silicona } \\
\text { Adhesivo en barra. }\end{array}$ \\
\hline
\end{tabular}

Con la finalidad de poder medir la satisfacción o percepción de las alumnas respecto del cuidado de los recursos naturales, en este caso particular, el agua, se realizó una actividad, en donde participaron alumnas de la Escuela Dama Blanca de Talcahuano. La actividad consistió en una charla de inmersión, luego una colación (un jugo en caja y 2 yogures), cuyos envases, luego de lavarlos bien, se utilizarían como material para construir sus propias propuestas para abordar la problemática y pudieran diseñar productos que ayuden a la extracción y manejo del agua. A través de un enfoque pragmático las alumnas tuvieron que "idear" sobre la marcha las propuestas, experimentando y modificando los volúmenes de los 2 envases de yogurt: Polietileno de alta densidad $(5 \mathrm{~cm} \times 5 \mathrm{~cm} \times 6 \mathrm{~cm})$, un envase de jugo: tetra brik de $(12 \mathrm{~cm} \times 2.5 \mathrm{~cm} \times 3 \mathrm{~cm})$. 
Ambos productos son muy accesibles a la población de estudio, por lo que constituye una buena manera para generar conciencia sobre el impacto del consumo en la sustentabilidad. Además a cada grupo se les entregó tijeras, cinta de enmascarar, silicona y adhesivo en barra. El objetivo principal de esta actividad fue indagar, en la importancia que las alumnas dan a los contenidos de reciclaje o reutilización para ayudar a la sustentabilidad; si la ciencia ficción, como recurso narrativo, puede ayudar a contextualizar prospectivamente una problemática, además de ayudar a inspirar y motivar a las alumnas a implicarse en la solución de problemáticas globales relacionadas, en este caso, con el recurso del agua. Se aplicó un muestreo no probabilístico, muestreo intencional o de conveniencia, para escoger el colegio en el que se realizaría la actividad y posterior aplicación de la encuesta en estudio, esto porque se requería un establecimiento que estuviera certificado en educación con respecto al cuidado del agua, por lo que la empresa ESSBIO, estableció que el colegio que reunió las características necesarias fue la Escuela Dama Blanca D-483, ubicada en calle Sargento Aldea 132, Talcahuano. Una vez escogida la escuela, para que participe de la Primera Feria Multimodal, se debe establecer qué alumnas o cursos concurrirán a dicha actividad. Es por ello que los profesores y encargados del establecimiento indicaron los cursos que cumplen con requisitos para este procedimiento, por lo que nuevamente se utilizó un muestreo no probabilístico. Finalmente la población objeto de estudio fueron alumnas de la Escuela Dama Blanca que hayan cursado la asignatura de Educación Tecnológica y que hubieran asistido a la Primera Feria Multimodal.

\section{Segunda etapa: Estudio Exploratorio}

Se realizó un estudio exploratorio (Creswell, 2003) con el objetivo de conocer la opinión respecto a la actividad desarrollada. La población de estudio consideró a las alumnas del sexto año (A), séptimo (C) y octavo año (A y B) con un total de 106 alumnas todas mujeres entre 12 y 14 años de edad. Se utilizó una encuesta de percepción mediante un cuestionario auto-administrado de 10 preguntas. 4 de las cuales evaluaban en términos generales el grado de importancia de la sustentabilidad y la administración de recursos, el reciclaje y reutilización como estrategias medio ambientales, la creatividad para solucionar problemas y la ciencia ficción, 4 preguntas apuntaron a conocer sus apreciaciones sobre la experiencia en general, ante las cuales las estudiantes debían responder según su grado de identificación con lo enunciado, empleando una escala Likert de cinco alternativas, desde 1 ("muy en desacuerdo") a 5 ("muy de acuerdo"). Finalmente se consideraron 2 preguntas abiertas que hacían referencia a los aspectos que más y menos les habían gustado: la inventiva y creatividad para solucionar problemas reales, la importancia de una visión holística, multidisciplinaria para diseñar soluciones y la posibilidad de estudiar carreras creativas /tecnológicas (Diseño industrial). La encuesta se aplicó previo procedimiento de Consentimiento Informado. Para el análisis de datos se realizó un análisis estadístico descriptivo de las respuestas de las alumnas participantes.

\section{RESULTADOS Y DISCUSIÓN}

A continuación se exponen los resultados obtenidos. En primer lugar se ilustran a través de registro fotográfico los tres momentos principales de la actividad: Exposición, Desafió y Premiación. En la figura 4 podemos observar instancias donde uno de los docentes explica el funcionamiento de las naves y su labor dentro del planeta marte. Junto con ello se pueden observar a las alumnas interactuando y contemplando las gigantografías y los dioramas.

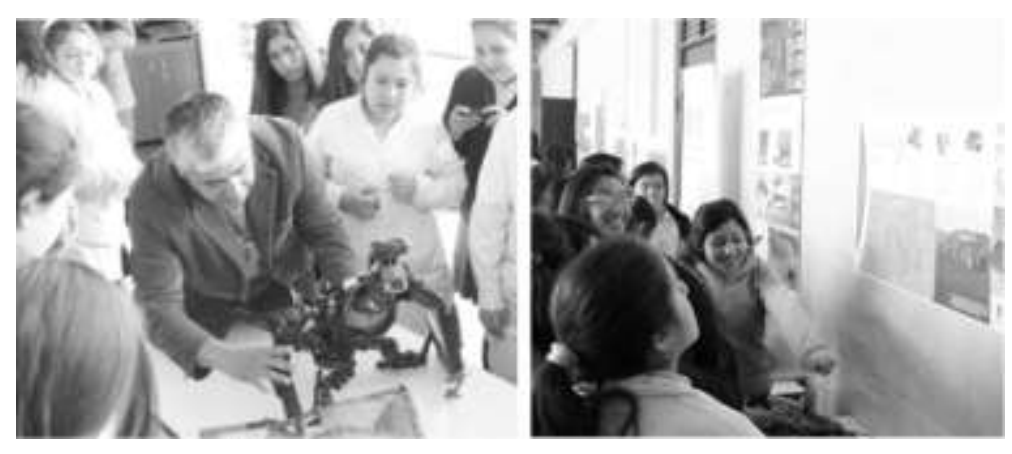

Fig. 4: Exposición y descripción de naves e infografías.

En la figura 5 podemos observar el proceso creativo /constructivo llevado por las alumnas durante el desarrollo del desafío. Una vez que se terminó de dar el encargo, las estudiantes tuvieron que agruparse y desarrollar en 45 minutos.

Finalmente los resultados de dicho desarrollo culminaron en la premiación. Instancia donde las alumnas tuvieron que exponer y presentar las características y el propósito del producto que desarrollaron. En las 
figura 6 podemos observar distintas propuestas relacionada con la tipología de vehículos. Desde carros colectores, naves exploradoras, hasta robots de inspección marciana. Junto con esto en la tabla 1 se describe el plan de actividades junto con los resultados obtenidos de cada una de ellas. En la primera columna se definen los aspectos claves que se consideraron en su desarrollo y en la segunda se describen los resultados.
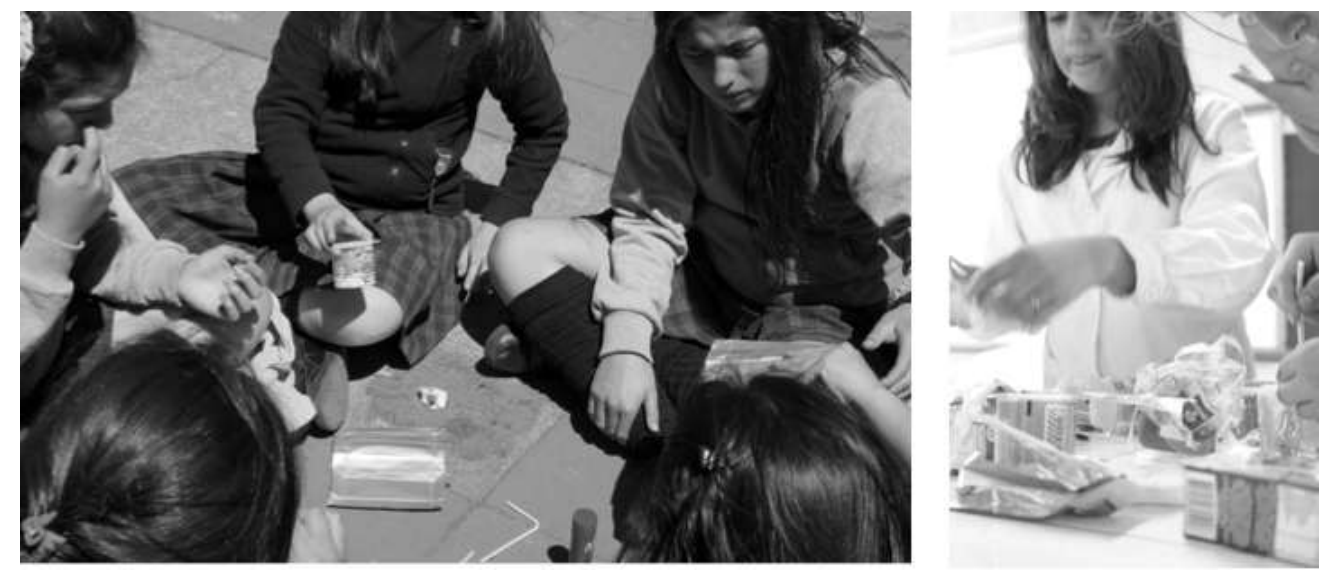

Fig. 5: Desafío. Grupos de alumnas construyendo propuestas reutilizando envases de deshecho.

Tabla 1: Plan de actividades y resultados obtenidos durante la intervención.

\begin{tabular}{|c|c|}
\hline Actividad & $\begin{array}{l}\text { Feria Educativa Multimodal Itinerante para el desarrollo de las habilidades creativas y } \\
\text { el pensamiento sustentable en niños de enseñanza básica y media de la } 8^{\mathrm{a}} \text { Región. }\end{array}$ \\
\hline Objetivo de la Actividad & $\begin{array}{l}\text { Estimular la creatividad desde la sustentabilidad a través de una experiencia } \\
\text { multimodal basada en la ciencia ficción. }\end{array}$ \\
\hline Metodología del Diseño & $\begin{array}{l}\text { Metodología basada en una inducción en narrativa de ciencia ficción, creatividad y una } \\
\text { concientización respecto de los problemas ambientales, encargo y creación de un } \\
\text { diseño futurista, participante de la narración, a partir de la reutilización y reciclaje de } \\
\text { los elementos de desecho de sus colaciones. }\end{array}$ \\
\hline \multirow{2}{*}{$\begin{array}{l}\text { Inmersión (Observación en } \\
\text { contexto) } \\
\text { Exposición de Tema }\end{array}$} & $\begin{array}{l}\text { Exposición, recorrido exploratorio, observación multi-sensorial de la exposición } \\
\text { (Láminas de infografía), Maquetas de naves reales a escala. }\end{array}$ \\
\hline & Escucha y percepción activa. \\
\hline $\begin{array}{l}\text { Planteamiento de la } \\
\text { Problemática }\end{array}$ & Escucha activa por parte de las alumnas. \\
\hline Encargo de Diseño & $\begin{array}{l}\text { Se definen las características, requisitos y condicionantes del encargo. Tipo de } \\
\text { producto que diseñar, objetivo y propósito de diseño. Usabilidad, etc. }\end{array}$ \\
\hline $\begin{array}{l}\text { Ideación, exploración creativa } \\
\text { de soluciones. }\end{array}$ & $\begin{array}{l}\text { Trabajo en Equipo: Se plantean las primeras ideas de soluciones utilizando bocetos } \\
\text { esquemáticos (Síntesis individual). } \\
\text { Estas ideas se analizan y se retroalimentan en forma grupal (Análisis grupal). } \\
\text { Seleccionando la idea que se va a desarrollar. }\end{array}$ \\
\hline $\begin{array}{l}\text { Proceso de reutilización } \\
\text { Exploración constructiva }\end{array}$ & $\begin{array}{l}\text { Se limpian los envases de yogur y jugo y se preparan para ser utilizados como } \\
\text { insumos en la construcción de la propuesta de diseño. } \\
\text { Se intervienen mecánicamente (cortes, pliegues, etc.) y se van conformando como } \\
\text { partes y piezas del producto propuesto. } \\
\text { Análisis y Lógica constructiva: selección y adaptación, qué pieza puede calzar, y } \\
\text { formar parte de, por ejemplo: “...tapa de envase podría ser carcasa de la nave". }\end{array}$ \\
\hline Presentación de propuestas. & $\begin{array}{l}\text { Pensamiento reflexivo, discurso y comunicación en público de características de la } \\
\text { propuesta. } \\
\text {..Nosotras pensamos en una cámara de fotos que pueda tomar fotos con sensores } \\
\text { para detectar el agua en marte, además de enviarlas a los otros investigadores que } \\
\text { están en la nave... }\end{array}$ \\
\hline Valoración y selección & $\begin{array}{l}\text { Una vez que se presentaron todas las propuestas se procede a elegir mediante } \\
\text { aplausos las más relevantes. }\end{array}$ \\
\hline $\begin{array}{l}\text { Productos o evidencias del } \\
\text { diseño }\end{array}$ & $\begin{array}{l}\text { Maqueta de producto realizada con base en la reutilización de envases de yogur y } \\
\text { jugos. }\end{array}$ \\
\hline
\end{tabular}



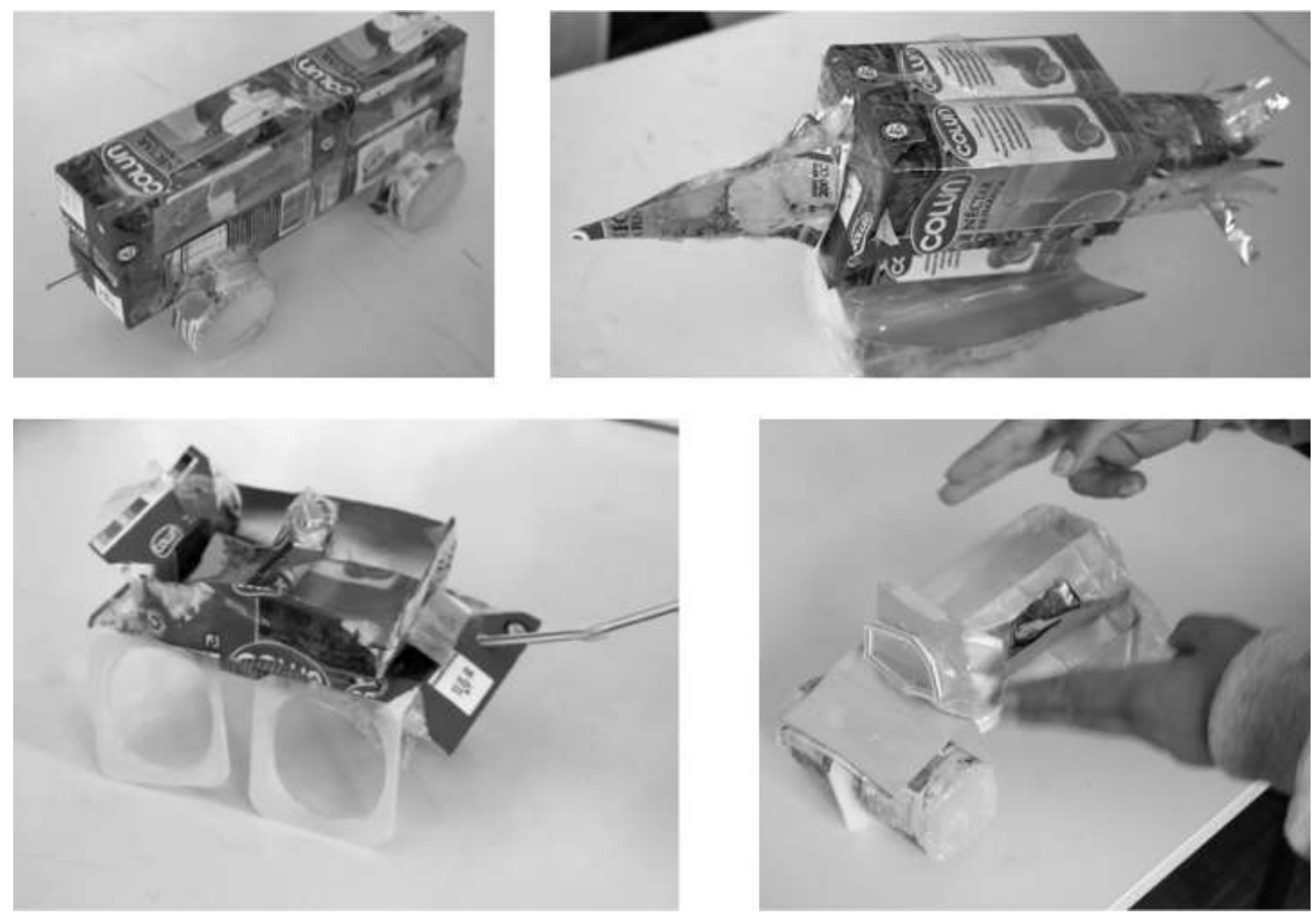

Fig. 6: Presentación de propuestas y premiación.

\section{Resultados del Estudio Exploratorio}

A continuación se desglosa la información referente a las 10 preguntas que se aplicaron en la Encuesta de Percepción, se muestran los gráficos asociados a las 4 primeras preguntas en la tabla 2 seguido de los resultados de las preguntas y la tabla 3 con el resumen de las respuestas abiertas.

Tabla 2: Resumen de resultados en torno a la sustentabilidad, creatividad, y ciencia ficción.

\begin{tabular}{|c|c|}
\hline $\begin{array}{l}\text { Pregunta 1: ¿Crees que la sustentabilidad es importante } \\
\text { a la hora de administrar los recursos naturales como el } \\
\text { agua? }\end{array}$ & $\begin{array}{l}\text { Pregunta 2: ¿Crees que la reutilización y reciclaje pueden } \\
\text { solucionar problemas a nivel mundial? }\end{array}$ \\
\hline $\begin{array}{l}\text { - Muy en desacuer do } \\
\text { " En desaciacrdo } \\
\text { Windiferente } \\
\text { - De acuerdo } \\
\text { "Muy de acuendo } \\
\text { " No Responde }\end{array}$ & $\begin{array}{l}\text { - Muy en desaruerdo } \\
\text { = En desacuerdo } \\
\text { = inditerente } \\
\text { = De acuerdo } \\
\text { = Muy de acuerdo } \\
\text { " No Responide }\end{array}$ \\
\hline $\begin{array}{l}\text { Pregunta 3: ¿Crees que la creatividad es importante para } \\
\text { solucionar problemas difíciles? }\end{array}$ & $\begin{array}{l}\text { Pregunta 4: ¿Crees que la ciencia ficción se puede } \\
\text { convertir en realidad? }\end{array}$ \\
\hline $\begin{array}{l}\text { "Mur en desacuerdo } \\
\text { "En desacuerdo } \\
\text { " Indiferente } \\
\text { - De acuerdo } \\
\text { "Muy de acuerdo } \\
\text { " No Responde }\end{array}$ & $\begin{array}{l}\text { Muy en desacuerdo } \\
\text { "En desacuerdo } \\
\text { a indiferente } \\
\text { - De acuerdo } \\
\text { - Muy de acuerdo } \\
\text { - No Responde }\end{array}$ \\
\hline
\end{tabular}


Del total de los encuestados, la mayoría, 97\%, está en parte y gran parte de acuerdo en que la sustentabilidad es importante a la hora de administrar los recursos naturales, como por ejemplo el agua. Mientras que los que no están de acuerdo o se sienten indiferentes a esta afirmación corresponden a un 3\% de la población. De las alumnas encuestadas, el $81 \%$ está muy de acuerdo y un $16 \%$ de acuerdo, en que la reutilización y reciclaje pueden solucionar problemas a nivel mundial; mientras que un $3 \%$ se muestra indiferente a esta pregunta. De las 106 alumnas encuestadas, el $73 \%$ está muy de acuerdo y el $19 \%$ de acuerdo en que la creatividad es importante para solucionar problemas difíciles, mientras que un $1 \%$ se muestra en desacuerdo y un $5 \%$ indiferente; el restante $2 \%$ no responde está pregunta.

La percepción de las alumnas encuestadas indica que un 58\% está muy de acuerdo y un $22 \%$ de acuerdo en que la ciencia ficción se puede convertir en realidad, mientras que un $4 \%$ está en desacuerdo y un $5 \%$ muy en desacuerdo con esta afirmación. El 11\% restante permanece indiferente a la pregunta.

El $89 \%$ de las alumnas cree que la experiencia de dicho programa fue muy buena, un $8 \%$ le pareció bueno, mientras que el 3\% de las encuestadas se mantuvo indiferente. El 79\% indicó que la narración-historia y los recursos multimodales (gigantografías, dioramas, videos) contribuyeron de manera categórica a la inmersión y concientización respecto a la problemática planteada. El $16 \%$ cree que fue buena y tuvo bastante injerencia, sin embargo el $1 \%$ está en desacuerdo y muy desacuerdo, para cada una. Además el $3 \%$ restante se muestra indiferente a esta pregunta.

Más del $80 \%$ de las alumnas está muy de acuerdo y un 10\% está de acuerdo en que le gustó participar de dicha actividad, mientras que un $1 \%$ está en desacuerdo y un $4 \%$ se muestra indiferente a esta pregunta. Además el $1 \%$ restante no responde. Y casi el $80 \%$ estaría muy de acuerdo en volver a realizar la misma experiencia.

Análisis de Correlación:

El análisis de correlación aplicado demostró que hay dependencia entre algunas de las preguntas, lo que quiere decir que la respuesta de una afecta en cierto grado la respuesta de la otra. En este caso la correlación es alta y positiva, por lo que para el objetivo principal de la encuesta, es favorable.

Análisis de Correspondencia Múltiple:

El análisis de correspondencia arrojó que la respuesta de las encuestadas, en su gran mayoría, se asemeja, de esta manera corroboró los resultados obtenidos en el análisis de correlación, al determinar que las ocho preguntas se podían expresar en dos dimensiones. Esto indica que existe algún patrón de relación entre cada una de las preguntas que estaban en las dimensiones 1 y 2.

En cuanto a la opinión de las alumnas recolectada en los datos cualitativos, los resultados se sintetizan en la Tabla 3, y evidencian una evaluación positiva de la experiencia, y de su correlación de la creatividad y su aplicación en carreras proyectuales creativas como el diseño industrial, expandiendo de posibles ofertas relacionadas con la formación profesional.

Tabla 3: Opinión de los estudiantes en la fase cualitativa

\begin{tabular}{|l|l|}
\hline \multicolumn{1}{|c|}{ Aspecto } & \multicolumn{1}{c|}{ Opinión } \\
\hline $\begin{array}{l}\text { La inventiva y creatividad para } \\
\text { solucionar problemas reales. }\end{array}$ & $\begin{array}{l}\text { La gran mayoría de las alumnas consideró que es muy importante ser creativos e } \\
\text { imaginativos al momento de solucionar problemas reales. La ciencia ficción generó } \\
\text { un contexto de fantasía que estimuló y motivó el desafío de poder trabajar a partir } \\
\text { de deshechos y transformarlos en algo útil. }\end{array}$ \\
\hline $\begin{array}{l}\text { Estudiar carreras creativas y o } \\
\text { tecnológicas (Diseño } \\
\text { industrial) }\end{array}$ & $\begin{array}{l}\text { La mitad de las alumnas se motivan a considerar seguir carreras } \\
\text { creativas/tecnológicas donde puedan desarrollar soluciones de impacto regional y } \\
\text { global. }\end{array}$ \\
\hline
\end{tabular}

\section{CONCLUSIONES}

La importancia de abordar los problemas ambientales y de sustentabilidad, como es el cuidado del recurso agua través de una intencionalidad pedagógica que utiliza la ciencia ficción como recurso narrativo y la reutilización/reciclaje de deshechos para la generación de propuestas de diseño demostró ser altamente efectiva en esta experiencia. El alto porcentaje de respuestas positivas, indica que las alumnas están de acuerdo que la sustentabilidad es importante a la hora de administrar recursos naturales como lo es el agua, así también en el reciclaje y la creatividad para solucionar problemas difíciles, además están de acuerdo en que la ciencia ficción se puede convertir en realidad. 
Se percibe como positivo la integración de una narración con recursos multimodales que permitan contextualizar la temática de estudio, para así captar y mantener la atención de las alumnas y lograr un impacto que permita un grado de sensibilización al respecto. Los resultados de la encuesta apoyan esta hipotesis, al hacer énfasis en el aporte de la ciencia ficción al desafío propuesto. Se observa además que las alumnas encuestadas estarían dispuestas a repetir la actividad, lo que refeja su satisfacción con la misma. Respecto de las materias tratadas en el taller, un amplio porcentaje de las encuestadas demostró un compromiso con el medio ambiente y con el reciclaje.

Si bien las alumnas cursaban los últimos años de Educación Básica, esta experiencia les permitió ampliar sus conocimientos respecto a los campos de aplicación del diseño, más allá de su rol estético/formal valorando su contribución a solucionar problemáticas actuales y futuras de la humanidad.

El ejercicio creativo expone sin dificultad, no sólo lo motivador, integrador, comprometido y efectivo que resulta como método didáctico, sino que además les permite a los estudiantes dimensionar la relevancia del actuar creativo como pensamiento tendiente a resolver problemas de importancia mundial. La valoración de los trabajos también aporta a la autoestima y fomenta un actuar consecuente a lo tratado teóricamente, es decir, convierte en significativo lo visto en clases. Curiosamente, pese a todas las virtudes que tiene un ejercicio como éste-que es sólo un ejemplo de las múltiples actividades creativas y multimodales que se pueden realizar en el aula- no resultan comunes, y eso sucede principalmente porque aún en nuestra cultura son los profesores y no los alumnos quienes poseen el rol protagónico en los espacios escolares.

\section{AGRADECIMIENTOS}

Los autores agradecen a la dirección de Investigación de la Universidad del Bío-Bío a través de su fondo FAPEI y a la Dirección de Extensión por haber financiado el proyecto de extensión código PER-06-2012.

\section{REFERENCIAS}

Bassett, C., E. Steinmueller y G. Voss, Better Made Up: The Mutual Influence of Science fiction and Innovation, (2013)

Binaa, O., S. Mateusc, L. Pereirab y A. Caffab, The future imagined: Exploring fiction as a means of reflecting on today's Grand Societal Challenges and tomorrow's options, http://dx.doi.org/10.1016/j.futures.2016.05.009, Futures (2016)

Birch, A., K.K.B. Hon y T. Short, Structure and output mechanisms in Design for Environment (DfE) tools, Journal of Cleaner Production, 35, 50 - 58 (2012)

Birkhofer, H., The Future of Design Methodology. Springer-Verlag London, Chapter 23 Design Research and Education, A University Perspective, D. Marjanovic (2011)

Blythe M.A. y P.C. Wright, Pastiche scenarios: Fiction as a resource for user centred design, Interacting with computers, 18 (5), 1139-1164 (2006)

Braha D. y O. Maimon, The Design Process: Properties, Paradigms, and Structure, IEEE Transactions on Systems, Man, and Cybernetics - Part A, Systems and Humans, 27, 2 (1997)

Brake, M. y R., Thornton, Science fiction in the classroom, Physics Education, 38 (1), 31 -34 (2003)

Braungart, M. y W. McDonough, De la Cuna a la Cuna, Rediseñando la Forma en que Hacemos las Cosas, McGraw- Hill Interamericana, Madrid, España, 190 pp. (2005)

Brezet, H. y C. Van Hemel, Ecodesign: a promising approach to sustainable production and consumption, Industry and environment, 20 (1 and 2), 52 (1997)

Capuz, S., T. Gomez, J. Vivancos, R. Viñoles, P. Ferrer, R. López y M. Bastante, Ecodiseño: Ingeniería del ciclo de vida para el desarrollo de productos sostenibles, Editorial UPV, Universidad Politécnica de Valencia, (2002)

Chankrajang, T. y R. Muttarak, Green Returns to Education: Does Schooling Contribute to ProEnvironmental Behaviours? Evidence from Thailand, Ecological Economics, 131, 434-448 (2017)

Creswell, J., Research Design, Qualitative, Quantitative and Mixed Methods Approaches, Thousand Oaks CA, Sage Publications SA. (2003) 
Cuello, A. Problemas Ambientales y Educación ambiental en la escuela. Centro Nacional de Educación Ambiental. Documento de trabajo para la Estrategia Andaluza de Educación Ambiental (en línea), http://www.mapama.gob.es/es/ceneam/articulos-de-opinion/2003_03cuello_tcm7-53015.pdf (2003)

Diego-Mas, J.A., R. Poveda-Bautista y J. Alcaide-Marzal, Designing the appearance of environmentally sustainable products, Journal of Cleaner Production, (135) 784-793 (2016)

Donovan, D., How children represent sustainable consumption through participatory action research and codesign of visual narratives, Journal of Consumer Studies, (5) 562 - 574 (2016)

Jones, C., Métodos de Diseño, Colección GG Diseño, Editorial Gustavo Gili, Barcelona (1977)

Laprise, S. y C. Winrich, The impact of science fiction films on student interest in science, Journal of College Science Teaching, 4 (2), 45-49 (2010)

Manzini, E., La materia de la invención, Materiales y proyectos, Ediciones CEAC, S.A. Biblioteca (1993)

McCright, A.M., The effects of gender on climate change knowledge and concern in the American public, Popul. Environ, 32, 66-87 (2010)

Moore, C. L., Technology development for human exploration of Mars, Acta Astronautica, (67) 1170-1175 (2010)

Olsson, D. y N. Gericke, The adolescent dip in students' sustainability consciousness-Implications for education for sustainable development, Journal of Environmental Education, 47(1), 35-51 (2016)

Pála B. y Á. Kereszturib, Possibility of microscopic liquid water formation at landing sites on Mars and their observational potential, Icarus 282, 84-92 (2017)

Robinson, D., L. Huang, Y. Guo y A.L. Porter, Forecasting Innovation Pathways (FIP) for new and emerging science and technologies, Technological Forecasting and Social Change, 80(2), 267-285 (2013)

Stern, P. C., Information, incentives, and proenvironmental consumer behavior, Journal of Consumer Policy, 22(4), 461-478 (1999)

Thoring, K., y R. M. Mueller, Fewer constraints more creativity? Insights from an educational science fiction project. The $2^{\text {nd }}$ International Conference on Design Creativity (ICDC2012) Glasgow, UK, 18 th $^{\text {t } 20^{\text {th }}}$ September, 307-316 (2012)

Tyla B., I. Lizarraldeb y R. Allaisc, Local value creation and eco-design: A new paradigm, Procedia CIRP 30 $155-160$ (2015)

Vallet, F., B. Eynard, D. Millet, S.G. Mahut, B. Tyl y G. Bertoluci ,Using eco-design tools: an overview of experts' practices, Design Studies, 34 (3), 345-377 (2013)

Woelfel C., J. Krzywinski y F. Drechsel, Knowing, Reasoning and Visualizing in Industrial Design, The Knowledge Engineering Review, (00)0, 11-99 (2010) 
